\title{
ANALYTICAL SOLUTIONS FOR A GENERAL MIXED BOUNDARY VALUE PROBLEM ASSOCIATED WITH MOTIONS OF FLUIDS WITH LINEAR DEPENDENCE OF VISCOSITY ON THE PRESSURE
}

\author{
D. VIERU* \\ Technical University of Iasi \\ ROMANIA \\ E-mail: dumitru_vieru@yahoo.com \\ C. FETECAU \\ Academy of Romanian Scientists, 050094 Bucharest \\ ROMANIA \\ C. BRIDGES \\ Department of Engineering, Texas Christian University \\ Fort Worth, TX-76129, USA
}

\begin{abstract}
An unsteady flow of incompressible Newtonian fluids with linear dependence of viscosity on the pressure between two infinite horizontal parallel plates is analytically studied. The fluid motion is induced by the upper plate that applies an arbitrary time-dependent shear stress to the fluid. General expressions for the dimensionless velocity and shear stress fields are established using a suitable change of independent variable and the finite Hankel transform. These expressions, that satisfy all imposed initial and boundary conditions, can generate exact solutions for any motion of this type of the respective fluids. For illustration, three special cases with technical relevance are considered and some important observations and graphical representations are provided. An interesting relationship is found between the solutions corresponding to motions induced by constant or ramptype shear stresses on the boundary. Furthermore, for validation of the results, the steady-state solutions corresponding to oscillatory motions are presented in different forms whose equivalence is graphically proved.
\end{abstract}

Key words: analytical solutions, mixed boundary value problem, pressure-dependent viscosity.

\section{Introduction}

The fact that the fluid viscosity depends on the pressure was already remarked in the seminal work of Stokes [1]. A justification of such an assumption comes from the fact that the viscosity can change by several orders of magnitude at significant variations of the pressure while the density remains almost unchanged. The manner in which the viscosity varies with the pressure has been both experimentally and theoretically studied and the most important results until 1931 can be found in the book of Bridgman [2]. Over time, a lot of theoretical and experimental studies regarding the variation of viscosity with the pressure have appeared but exact solutions for motions of fluids with pressure-dependent viscosity are very few in the existing literature. On the other hand, in all studies prior to 2004, the effect of gravity on the flows of such fluids was neglected (see for instance Hron et al. [3]; Rajagopal and Szeri [4]; Vasudevaiah and Rajagopal [5]) although there are many important problems in which the phenomena under consideration essentially depend on the gravity action and the problem of Bernard convection is such an example.

\footnotetext{
${ }^{*}$ To whom correspondence should be addressed
} 
The first exact solutions for steady motions of fluids with pressure dependent viscosity in which the effect of gravity is taken into consideration seem to be those of Rajagopal [6]. A little later, Kannan and Rajagopal [7] studied the flow of such fluids between two parallel plates rotating about a common axis or different axes and found that gravity has a significant effect on the flow characteristics. Rajagopal [8] investigated the flow of a fluid with pressure dependent viscosity over an inclined plane and found that the vorticity variation is non-monotone. Numerical results regarding unsteady motions of such fluids were provided by Massoudi and Phuok [9] and Srinivasan and Rajagopal [10]. The first exact solutions for unsteady plane shearing flows of fluids with pressure dependent viscosity seem to be those of Rajagopal and Saccomandi [11]. Exact solutions for modified unsteady Stokes' problems with linear and exponential dependence of viscosity on the pressure have been established by Prusa [12] in terms of Kelvin functions but a more general and interesting study of the same problems was developed by Rajagopal et al. [13]. Other exact or numerical solutions for motions of fluids with pressure dependent viscosity in cylindrical or spherical domains have been also obtained by Kalogirou et al. [14], respectively Housiadas et al. [15].

It is worth pointing out the fact that all exact solutions that have been previously presented correspond to fluid motions in which the velocity is given on the boundary although in many practical problems the shear stress is specified on the boundary [Renardy 16, 17]. In these cases, contrary to what is usually assumed, prescribing the shear stress on the boundary is tantamount to prescribing the force to move it. Actually, in Newtonian mechanics, the force is the cause and kinematics is the effect. Furthermore, the noslip boundary condition may not be applicable for flows of polymeric fluids that can slip or slide on the boundary. To the best of our knowledge, the first exact solutions for fluid flows in which the shear stress is given on the boundary have been established by Waters and King [18]. In the last fifteen years, a lot of exact solutions for motions induced by shear stresses on the boundary have been published but none of them for fluids with pressure-dependent viscosities.

It is well known that the exact solutions play a double scope. In addition, to provide solutions for problems with technical relevance, they can be used as tests to verify numerical schemes that are developed to study flows of such fluids in complex geometries. The main purpose of this paper is to provide a general class of simple exact solutions for motions of incompressible Newtonian fluids with linear dependence of viscosity on the pressure between two infinite horizontal parallel plates. The fluid motion is generated by the upper plate that applies a time-dependent shear stress to the fluid. The general expressions that have been established for velocity and shear stress fields satisfy all imposed initial and boundary conditions and can generate exact solutions for any motion of this kind of respective fluids. For their validation, the solutions corresponding to oscillatory motions are presented in two different forms whose equivalence is graphically proved. A relationship between the solutions corresponding to constant or ramp-type shear stresses on the boundary is established and some graphical representations are presented and discussed.

\section{Constitutive and governing equations}

The constitutive equation of incompressible Newtonian fluids with pressure-dependent viscosity, as it results from Rajagopal $[6,8]$, is given by

$$
\boldsymbol{T}=-p \boldsymbol{I}+\eta(p) \boldsymbol{A} \quad \text { with } \quad \operatorname{tr} \boldsymbol{A}=0,
$$

where $\boldsymbol{T}$ is the Cauchy stress tensor, $-p \boldsymbol{I}$ is the reaction stress due to the constraint of incompressibility, $\boldsymbol{A}$ is the first Rivlin-Ericksen tensor and $\eta(p)$ is the fluid viscosity. Since the fluid viscosity increases for increasing values of the pressure $p, \eta(\cdot)$ is a positive and increasing function. In what follows, we shall consider a linear dependence of viscosity on the pressure, namely Rajagopal et al. [13, Eq. (4) with $n=1$ ]

$$
\eta(p)=\mu\left[\alpha\left(p-p_{h}\right)+1\right]
$$


where $\mu$ is the fluid viscosity at the reference pressure $p_{h}$ and $\alpha$ is a positive constant. In this form $\eta(p) \rightarrow \infty$ as $p \rightarrow \infty$, a property that has been experimentally proved.

Let us consider such a fluid at rest between two infinite horizontal parallel plates at the distance $h$ apart. At the moment $t=0^{+}$the upper plate begins to apply a time-dependent shear stress $S f(t)$ to the fluid. Here $S$ is a constant shear-stress while the dimensionless function $f(\cdot)$ is piecewise continuous and $f(0)=0$. Because of the shear the fluid is gradually moved and we are looking for a velocity field of the form

$$
\boldsymbol{v}=\boldsymbol{v}(y, t)=v(y, t) \mathbf{i}, \quad p=p(y, t)
$$

where $\mathbf{i}$ is the unit vector along the $x$-axis of a suitable Cartesian coordinate system $x, y$ and $z$ whose $y$-axis is perpendicular to the plates. The continuity equation is identically satisfied.

On the basis of Eqs (2.3), the constitutive Eq.(2.1) $)_{1}$ and the balance of linear momentum can be reduced to the relevant partial differential equations

$$
\mu \frac{\partial}{\partial y}\left\{\left[\alpha\left(p-p_{h}\right)+1\right] \frac{\partial v(y, t)}{\partial y}\right\}=\rho \frac{\partial v(y, t)}{\partial t}, \quad \frac{\partial p(y, t)}{\partial y}+\rho g=0
$$

where $\rho$ is the fluid density and $g$ is the acceleration due to gravity. From the second relation of Eqs (2.4) it results that

$$
p(y, t)=-\rho g y+c(t)
$$

where $c(\cdot)$ is an arbitrary function. Following Prusa [12] and Rajagopal et al. [13] we consider the case of a pressure field for which the function $c(\cdot)$ is constant and therefore

$$
p(y)=\rho g(h-y)+p_{h}
$$

where $p_{h}=p(h)$ is the reference pressure.

Substituting $p(y)$ from Eq. (2.6) in (2.4) $)_{1}$, we find the following governing equation

$$
\mu[\alpha \rho g(h-y)+1] \frac{\partial^{2} v(y, t)}{\partial y^{2}}-\mu \alpha \rho g \frac{\partial v(y, t)}{\partial y}=\rho \frac{\partial v(y, t)}{\partial t} ; \quad 0<y<h, \quad t>0
$$

for the velocity field $v(y, t)$. The corresponding non-trivial shear stress $\tau(y, t)$, as it results from Eqs (2.1)(2.3), is given by

$$
\tau(y, t)=\mu[\alpha \rho g(h-y)+1] \frac{\partial v(y, t)}{\partial y} ; \quad 0<y<h, \quad t>0 .
$$

At the initial moment the fluid velocity and the shear stress have to be zero, i.e.

$$
v(y, 0)=0, \quad \tau(y, 0)=0 ; \quad 0 \leq y \leq h .
$$

The appropriate boundary conditions are 


$$
v(0, t)=0, \quad \tau(h, t)=S f(t) ; \quad t>0 .
$$

Introducing the following non-dimensional variables and functions

$$
y^{*}=\frac{y}{h}, \quad t^{*}=\frac{t}{t_{0}}, \quad v^{*}=\frac{\mu}{h S} v, \quad \tau^{*}=\frac{\tau}{S}
$$

where $t_{0}$ is a characteristic time and dropping out the star notation, we get the next initial and boundary value problem

$$
\begin{aligned}
& (\delta+1-y) \frac{\partial^{2} v(y, t)}{\partial y^{2}}-\frac{\partial v(y, t)}{\partial y}=\frac{\partial v(y, t)}{\partial t} ; \quad 0<y<1, \quad t>0, \\
& v(y, 0)=0 ; \quad 0 \leq y \leq 1, \\
& v(0, t)=0,\left.\quad \frac{\partial v(y, t)}{\partial y}\right|_{y=1}=f(t) ; \quad t>0,
\end{aligned}
$$

for the dimensionless velocity field $v(y, t)$.

In order to get the partial differential Eqs (2.12) we chosen the characteristic time $t_{0}$ to be equal with $h /(\alpha g \mu)$. In addition, the dimensionless pressure-viscosity parameter $\delta=1 /(\alpha \rho g h)$ and the nondimensional shear stress $\tau(y, t)$ is given by the relation

$$
\tau(y, t)=\frac{\delta+1-y}{\delta} \frac{\partial v(y, t)}{\partial y} ; \quad 0<y<1, \quad t>0 .
$$

\section{Solution of the problem}

In order to solve the partial differential Eq.(2.12) with the initial and boundary conditions (2.13) and (2.14) we first make the change of the independent variable

$$
y=1+\delta-\frac{r^{2}}{4} \quad \text { or equivalently } \quad r=2 \sqrt{\delta+1-y}
$$

The governing Eq.(2.12) takes a suitable form, namely

$$
\frac{\partial^{2} v(r, t)}{\partial r^{2}}+\frac{1}{r} \frac{\partial v(r, t)}{\partial r}=\frac{\partial v(r, t)}{\partial t} ; \quad a<r<b, \quad t>0
$$

while the initial and boundary conditions (2.13) and (2.14) become

$$
v(r, 0)=0 ; \quad a \leq r \leq b,
$$




$$
\left.\frac{\partial v(r, t)}{\partial r}\right|_{r=a}=-\sqrt{\delta} f(t), \quad v(b, t)=0 ; \quad t>0
$$

where

$$
a=2 \sqrt{\delta} \quad \text { and } \quad b=2 \sqrt{\delta+1} .
$$

In what follows, we use the finite Hankel transform defined by Debnath and Bhatta [19]

$$
v_{H n}(t)=\int_{a}^{b} r v(r, t) A\left(r, r_{n}\right) d r, \quad n=1,2,3 \ldots
$$

where

$$
A\left(r, r_{n}\right)=Y_{1}\left(a r_{n}\right) J_{0}\left(r r_{n}\right)-J_{l}\left(a r_{n}\right) Y_{0}\left(r r_{n}\right)
$$

$J_{0}(\cdot), Y_{0}(\cdot), J_{l}(\cdot)$ and $Y_{l}(\cdot)$ are standard Bessel functions of the first and second kind and $r_{n}$ are the positive roots of the transcendental equation

$$
A(b, r)=Y_{1}(a r) J_{0}(b r)-J_{1}(a r) Y_{0}(b r)=0 .
$$

Multiplying Eq.(3.2) by $r A\left(r, r_{n}\right)$, integrating the result with respect to $r$ from $a$ to $b$ and bearing in mind the identities (A1) and (A2) from the Appendix and the initial and boundary conditions (3.3) and (3.4), we find that

$$
\begin{aligned}
& \dot{v}_{H n}(t)+r_{n}^{2} v_{H n}(t)=-\frac{2 \sqrt{\delta}}{\pi r_{n}} f(t) ; \quad t>0, \\
& v_{H n}(0)=0 \quad \text { for each } \quad n=1,2,3 \ldots
\end{aligned}
$$

Now, solving the ordinary differential Eq.(3.8) with the initial condition (3.9) and introducing the result in the inverse finite Hankel transform given by Eq.(A3), we find that

$$
v(r, t)=-\pi \sqrt{\delta} \sum_{n=1}^{\infty} \frac{r_{n} J_{0}^{2}\left(b r_{n}\right)}{J_{l}^{2}\left(a r_{n}\right)-J_{0}^{2}\left(b r_{n}\right)} A\left(r, r_{n}\right) \int_{0}^{t} f(s) \mathrm{e}^{-r_{n}^{2}(t-s)} d s
$$

In this form the solution (3.10) satisfies the initial condition (3.3) and the boundary condition (3.4) 2 . In order to show that the boundary condition $(3.4)_{1}$ is also satisfied, we use the identity

$$
a \int_{a}^{b} r \ln \left(\frac{r}{b}\right) A\left(r, r_{n}\right) d r=\frac{2}{\pi r_{n}^{3}}
$$

and present the velocity field $v(r, t)$ in the equivalent form 


$$
v(r, t)=-2 \delta f(t) \ln \left(\frac{r}{b}\right)+\pi \sqrt{\delta} \sum_{n=1}^{\infty} \frac{J_{0}^{2}\left(b r_{n}\right) A\left(r, r_{n}\right)}{r_{n}\left[J_{l}^{2}\left(a r_{n}\right)-J_{0}^{2}\left(b r_{n}\right)\right]} \int_{0}^{t} f^{\prime}(s) \mathrm{e}^{-r_{n}^{2}(t-s)} d s .
$$

Coming back to the initial variables, we obtain for the dimensionless velocity field $v(y, t)$ under the suitable form

$$
v(y, t)=\delta f(t) \ln \left(\frac{\delta+1}{\delta+1-y}\right)+\pi \sqrt{\delta} \sum_{n=1}^{\infty} \frac{J_{0}^{2}\left(b r_{n}\right) A\left(2 \sqrt{\delta+1-y}, r_{n}\right)}{r_{n}\left[J_{l}^{2}\left(a r_{n}\right)-J_{0}^{2}\left(b r_{n}\right)\right]} \int_{0}^{\prime} f^{\prime}(s) \mathrm{e}^{-r_{n}^{2}(t-s)} d s
$$

which clearly satisfies all imposed initial and boundary conditions.

The adequate shear stress, as it results from Eqs (2.15) and (3.13), is given by

$$
\tau(y, t)=f(t)-\pi \frac{\sqrt{\delta+1-y}}{\sqrt{\delta}} \sum_{n=1}^{\infty} \frac{J_{0}^{2}\left(b r_{n}\right) B\left(2 \sqrt{\delta+1-y}, r_{n}\right)}{J_{1}^{2}\left(a r_{n}\right)-J_{0}^{2}\left(b r_{n}\right)} \int_{0}^{t} f^{\prime}(s) \mathrm{e}^{-r_{n}^{2}(t-s)} d s,
$$

where $B\left(r, r_{n}\right)=Y_{l}\left(a r_{n}\right) J_{l}\left(r r_{n}\right)-J_{l}\left(a r_{n}\right) Y_{l}\left(r r_{n}\right)$. The frictional force per unit area exerted by the fluid on the stationary plate is given by

$$
\tau_{0}(t)=\tau(0, t)=f(t)-\pi \sqrt{\frac{\delta+1}{\delta}} \sum_{n=l}^{\infty} \frac{J_{0}^{2}\left(b r_{n}\right) B\left(b, r_{n}\right)}{J_{l}^{2}\left(a r_{n}\right)-J_{0}^{2}\left(b r_{n}\right)} \int_{0}^{t} f^{\prime}(s) \mathrm{e}^{-r_{n}^{2}(t-s)} d s .
$$

Finally, it is worth pointing out the fact that the general expressions (3.13) and (3.14) can generate exact solutions for any motion with technical relevance of this type of incompressible Newtonian fluids with linear dependence of viscosity on the pressure. Consequently, the problem under discussion is completely solved and, in order to bring to light some physical insight of the obtained results, three special cases will be considered.

\subsection{Case $f(t)=H(t)$ (Upper plate applies a constant shear stress to the fluid)}

Replacing $f(t)$ by $H(t)$ (the Heaviside unit step function) in Eqs (3.13) and (3.14) and using the fact that the derivative of $H(t)$ is the Dirac delta function, we find the dimensionless velocity and shear stress fields

$$
\begin{aligned}
& v_{C}(y, t)=\delta H(t) \ln \left(\frac{\delta+1}{\delta+1-y}\right)+\pi \sqrt{\delta} H(t) \sum_{n=1}^{\infty} \frac{J_{0}^{2}\left(b r_{n}\right) A\left(2 \sqrt{\delta+1-y}, r_{n}\right)}{r_{n}\left[J_{l}^{2}\left(a r_{n}\right)-J_{0}^{2}\left(b r_{n}\right)\right]} \mathrm{e}^{-r_{n}^{2} t}, \\
& \tau_{C}(y, t)=H(t)-\pi H(t) \frac{\sqrt{\delta+1-y}}{\sqrt{\delta}} \sum_{n=1}^{\infty} \frac{J_{0}^{2}\left(b r_{n}\right) B\left(2 \sqrt{\delta+1-y}, r_{n}\right)}{J_{l}^{2}\left(a r_{n}\right)-J_{0}^{2}\left(b r_{n}\right)} e^{-r_{n}^{2} t},
\end{aligned}
$$

corresponding to the fluid motion induced by the upper plate that applies a constant shear stress $S H(t)$ to the fluid. The frictional force per unit area exerted by the fluid on the stationary plate, corresponding to this motion, is given by 


$$
\tau_{C_{0}}(t)=\tau_{C}(0, t)=H(t)-\pi H(t) \frac{\sqrt{\delta+1}}{\sqrt{\delta}} \sum_{n=1}^{\infty} \frac{J_{0}^{2}\left(b r_{n}\right) B\left(b, r_{n}\right)}{r_{n}^{2}\left[J_{l}^{2}\left(a r_{n}\right)-J_{0}^{2}\left(b r_{n}\right)\right]^{-r_{n}^{2} t}}
$$

Making $t \rightarrow \infty$ into Eqs (3.16) and (3.17) we find the steady (permanent) solutions

$$
v_{C p}(y)=\delta \ln \left(\frac{\delta+1}{\delta+1-y}\right), \quad \tau_{C p}(y)=1 .
$$

Consequently, in the steady case, the shear stress is constant on the whole flow domain although the fluid velocity is a function of $y$. This constant is just the constant shear stress applied by the upper plate to the fluid.

\subsection{Case $f(t)=t^{\alpha} H(t),(\alpha>0)$ (Upper plate applies ramp-type shear stress to the fluid)}

The solutions corresponding to the motion induced by the upper plate that applies a ramp-type (see Toki and Tokis [20]) shear stress to the fluid, namely

$$
\begin{aligned}
& v_{\alpha}(y, t)=\delta t^{\alpha} \ln \left(\frac{\delta+1}{\delta+1-y}\right)+\pi \alpha \sqrt{\delta} \sum_{n=1}^{\infty} \frac{J_{0}^{2}\left(b r_{n}\right) A\left(2 \sqrt{\delta+1-y}, r_{n}\right)}{r_{n}\left[J_{l}^{2}\left(a r_{n}\right)-J_{0}^{2}\left(b r_{n}\right)\right]} \int_{0}^{t}(t-s)^{\alpha-1} \mathrm{e}^{-r_{n}^{2} s} d s, \\
& \tau_{\alpha}(y, t)=t^{\alpha}-\pi \alpha \frac{\sqrt{\delta+1-y}}{\sqrt{\delta}} \sum_{n=1}^{\infty} \frac{J_{0}^{2}\left(b r_{n}\right) B\left(2 \sqrt{\delta+1-y}, r_{n}\right)}{J_{l}^{2}\left(a r_{n}\right)-J_{0}^{2}\left(b r_{n}\right)} \int_{0}^{t}(t-s)^{\alpha-1} \mathrm{e}^{-r_{n}^{2} s} d s
\end{aligned}
$$

are immediately obtained substituting $f(t)$ by $t^{\alpha} H(t)$ in Eqs (3.13) and (3.14). It is interesting to observe that the solutions corresponding to $\alpha=1$, namely

$$
\begin{aligned}
& v_{l}(y, t)=\delta t \ln \left(\frac{\delta+1}{\delta+1-y}\right)+\pi \sqrt{\delta} \sum_{n=1}^{\infty} \frac{J_{0}^{2}\left(b r_{n}\right) A\left(2 \sqrt{\delta+1-y}, r_{n}\right)}{r_{n}^{3}\left[J_{l}^{2}\left(a r_{n}\right)-J_{0}^{2}\left(b r_{n}\right)\right]}\left(1-e^{-r_{n}^{2} t}\right), \\
& \tau_{l}(y, t)=t-\pi \frac{\sqrt{\delta+1-y}}{\sqrt{\delta}} \sum_{n=1}^{\infty} \frac{J_{0}^{2}\left(b r_{n}\right) B\left(2 \sqrt{\delta+1-y}, r_{n}\right)}{r_{n}^{2}\left[J_{l}^{2}\left(a r_{n}\right)-J_{0}^{2}\left(b r_{n}\right)\right]}\left(1-e^{-r_{n}^{2} t}\right),
\end{aligned}
$$

can also be presented in simpler forms

$$
v_{l}(y, t)=\int_{0}^{t} v_{C}(y, s) d s \quad \text { and } \quad \tau_{l}(y, t)=\int_{0}^{t} \tau_{C}(y, s) d s
$$

where $v_{C}(y, t)$ and $\tau_{C}(y, t)$ are given by Eqs (3.16) and (3.17).

Furthermore, it is not difficult to show that Fetecau et al. [21] 


$$
v_{\alpha}(y, t)=\Gamma(\alpha+1) I_{t}^{\alpha}\left\{u_{C}(y, t)\right\}, \quad \tau_{\alpha}(y, t)=\Gamma(\alpha+1) I_{t}^{\alpha}\left\{\tau_{C}(y, t)\right\}
$$

where the Riemann-Liouville fractional integral operator $I_{t}^{\alpha}(\cdot)$ is defined by Hristov [22]

$$
I_{t}^{\alpha}\{f(t)\}=\frac{1}{\Gamma(\alpha)} \int_{0}^{t}(t-s)^{\alpha-1} f(s) d s
$$

where $\Gamma(\cdot)$ is the Gamma function. In the special case, when $\alpha=n$ (a natural number), Eqs (3.25) take the simpler forms

$$
\begin{aligned}
& v_{n}(y, t)=(n !) I_{t}^{n}\left\{v_{C}(y, t)\right\}=(n !) \int_{0}^{t} \int_{0}^{s_{l}} \int_{0}^{s_{2}} \ldots \int_{0}^{s_{n-1}} v_{C}\left(y, s_{n}\right) d s_{n} d s_{n-1} \ldots d s_{1}, \\
& \tau_{n}(y, t)=(n !) I_{t}^{n}\left\{\tau_{C}(y, t)\right\}=(n !) \int_{0}^{t} \int_{0}^{s_{l}} \int_{0}^{s_{2}} \ldots \int_{0}^{s_{n}} \tau_{C}\left(y, s_{n}\right) d s_{n} d s_{n-1} \ldots d s_{1} .
\end{aligned}
$$

Consequently, knowing the solutions $v_{C}(y, t)$ and $\tau_{C}(y, t)$ corresponding to the fluid motion due to a constant shear stress on the boundary, we can determine the adequate solutions for motions induced by the upper plate that applies shear stresses of the form $t^{\alpha} H(t)$ on the fluid using the Riemann-Liouville fractional integral operator $I_{t}^{\alpha}\{\}$ defined by Eq.(3.26). If $\alpha$ is a natural number $n$, the corresponding solutions can be obtained by simple or multiple integrations from $v_{C}(y, t)$, respectively $\tau_{C}(y, t)$ like in Eqs (3.27).

\subsection{Case $f(t)=H(t) \cos (\omega t)$ or $f(t)=H(t) \sin (\omega t)$ (Upper plate applies oscillatory shear stresses to the fluid)}

By substituting the function $f(t)$ by $H(t) \cos (\omega t)$ or $H(t) \sin (\omega t)$ in Eq.(3.13), where $\omega$ is the nondimensional frequency of the oscillations, we find the dimensionless velocity fields $v_{c}(y, t)$ and $v_{s}(y, t)$ corresponding to motions of incompressible Newtonian fluids with linear dependence of viscosity on the pressure induced by the upper plate that applies oscillatory share stresses to the fluid. Of course, these starting solutions can be presented as sums of steady-state (permanent) $v_{c p}(y, t)$ and $v_{s p}(y, t)$ and transient $v_{c t}(y, t)$ and $v_{s t}(y, t)$ components, namely

where

$$
v_{c}(y, t)=\left[v_{c p}(y, t)+v_{c t}(y, t)\right] H(t), \quad v_{s}(y, t)=\left[v_{s p}(y, t)+v_{s t}(y, t)\right] H(t)
$$

$$
\begin{aligned}
& v_{c p}(y, t)=\delta \ln \left(\frac{\delta+1}{\delta+1-y}\right) \cos (\omega t)+ \\
& -\omega \pi \sqrt{\delta} \sum_{n=1}^{\infty} \frac{J_{0}^{2}\left(b r_{n}\right) A\left(2 \sqrt{\delta+1-y}, r_{n}\right)}{J_{l}^{2}\left(a r_{n}\right)-J_{0}^{2}\left(b r_{n}\right)} \frac{r_{n}^{2} \sin (\omega t)-\omega \cos (\omega t)}{r_{n}\left(r_{n}^{4}+\omega^{2}\right)},
\end{aligned}
$$




$$
\begin{aligned}
& v_{c t}(y, t)=\pi \sqrt{\delta} \sum_{n=1}^{\infty} \frac{J_{0}^{2}\left(b r_{n}\right) A\left(2 \sqrt{\delta+1-y}, r_{n}\right)}{J_{l}^{2}\left(a r_{n}\right)-J_{0}^{2}\left(b r_{n}\right)} \frac{r_{n}^{3}}{r_{n}^{4}+\omega^{2}} \mathrm{e}^{-r_{n}^{2} t}, \\
& v_{s p}(y, t)=\delta \ln \left(\frac{\delta+1}{\delta+1-y}\right) \sin (\omega t)+ \\
& +\omega \pi \sqrt{\delta} \sum_{n=1}^{\infty} \frac{J_{0}^{2}\left(b r_{n}\right) A\left(2 \sqrt{\delta+1-y}, r_{n}\right)}{J_{l}^{2}\left(a r_{n}\right)-J_{0}^{2}\left(b r_{n}\right)} \frac{r_{n}^{2} \cos (\omega t)+\omega \sin (\omega t)}{r_{n}\left(r_{n}^{4}+\omega^{2}\right)}, \\
& v_{s t}(y, t)=-\omega \pi \sqrt{\delta} \sum_{n=1}^{\infty} \frac{J_{0}^{2}\left(b r_{n}\right) A\left(2 \sqrt{\delta+1-y}, r_{n}\right)}{J_{l}^{2}\left(a r_{n}\right)-J_{0}^{2}\left(b r_{n}\right)} \frac{r_{n}}{r_{n}^{4}+\omega^{2}} \mathrm{e}^{-r_{n}^{2} t} .
\end{aligned}
$$

An important remark regarding these solutions refers to the fact that, as expected, the velocity field $v_{c}(y, t)$ tends to the solution $v_{C}(y, t)$ when the oscillations' frequency $\omega \rightarrow 0$.

For a check of the obtained results, let us determine new but equivalent expressions for the steadystate components $v_{c p}(y, t)$ and $v_{s p}(y, t)$ of the starting solutions $v_{c}(y, t)$ and $v_{s}(y, t)$. To do that, we denote by $u_{p}(r, t)$ the complex velocity

$$
u_{p}(r, t)=v_{c p}(r, t)+i v_{s p}(r, t)
$$

where $v_{c p}(r, t)$ and $v_{s p}(r, t)$ are the steady-state components of the starting solutions of the initial and boundary value problem (3.2)-(3.4) and $i$ is the imaginary unit. Of course, $u_{p}(r, t)$ has to satisfy the partial differential equation

$$
\frac{\partial^{2} u_{p}(r, t)}{\partial r^{2}}+\frac{l}{r} \frac{\partial u_{p}(r, t)}{\partial r}=\frac{\partial u_{p}(r, t)}{\partial t} ; \quad a<r<b, \quad t \in R,
$$

with the initial conditions

$$
\left.\frac{\partial u_{p}(r, t)}{\partial r}\right|_{r=a}=-\sqrt{\delta} \mathrm{e}^{i \omega t}, \quad v(b, t)=0 ; \quad t \in R .
$$

For the boundary value problem (3.34)-(3.35) we are looking for a separable solution

$$
u_{p}(r, t)=V(r) F(t)
$$

Substituting Eq.(3.36) in Eq.(3.34) and bearing in mind the boundary conditions (3.35), we find that

$$
V^{\prime \prime}(r)+\frac{1}{r} V^{\prime}(r)-i \omega V(r)=0 ; \quad V^{\prime}(a)=-\sqrt{\delta}, \quad V(b)=0
$$

The general solution of Eq.(3.37) 1 , namely 


$$
V(r)=C_{1} I_{0}(r \sqrt{i \omega})+C_{2} K_{0}(r \sqrt{i \omega})
$$

is immediately obtained using the change of variable $r=s / \sqrt{i \omega}$. On the basis of boundary conditions $(3.37)_{2}$ and (3.37) $)_{3}$ we can determine the constants $C_{1}$ and $C_{2}$ and then the new expressions of the steadystate solutions $v_{c p}(r, t)$ and $v_{s p}(r, t)$. Coming back to the original variable, we found the simpler expressions

$$
\begin{aligned}
& v_{c p}(y, t)=\sqrt{\delta} \operatorname{Re}\left\{\frac{K_{0}(b \sqrt{i \omega}) I_{0}[2 \sqrt{i \omega(\delta+1-y)}]-I_{0}(b \sqrt{i \omega}) K_{0}[2 \sqrt{i \omega(\delta+1-y)}]}{I_{0}(b \sqrt{i \omega}) K_{0}^{\prime}(a \sqrt{i \omega})-K_{0}(b \sqrt{i \omega}) I_{0}^{\prime}(a \sqrt{i \omega})} \frac{\mathrm{e}^{i \omega t}}{\sqrt{i \omega}}\right\}, \\
& v_{s p}(y, t)=\sqrt{\delta} \operatorname{Im}\left\{\frac{K_{0}(b \sqrt{i \omega}) I_{0}[2 \sqrt{i \omega(\delta+1-y)}]-I_{0}(b \sqrt{i \omega}) K_{0}[2 \sqrt{i \omega(\delta+1-y)}]}{I_{0}(b \sqrt{i \omega}) K_{0}^{\prime}(a \sqrt{i \omega})-K_{0}(b \sqrt{i \omega}) I_{0}^{\prime}(a \sqrt{i \omega})} \frac{\mathrm{e}^{i \omega t}}{\sqrt{i \omega}}\right\}
\end{aligned}
$$

where Re and Im denote the real, respectively the imaginary part of that which follows. Figures $1 \mathrm{a}$ and $1 \mathrm{~b}$ clearly show that expressions (3.29) and (3.31) of the steady-state components $v_{c p}(y, t)$ and $v_{s p}(y, t)$ are equivalent to those given by relations (3.39), respectively (3.40).

It is well know that the steady-state solutions are independent of the initial conditions but they satisfy governing equations and boundary conditions. In practice, they are important for those who want to eliminate the transients from their experiments. For that one needs to know the time after which the fluid flows according to the steady-state solutions. This is the time after which the diagrams of starting solutions are almost identical to those of their steady-state components and it can be graphically determined as shown in following by Fetecau and Agop [21].

\section{Numerical results and discussions}

An analytical study of the unsteady flows of incompressible Newtonian fluids with linear dependence of viscosity on the pressure in a rectangular channel was carried out. The fluid motion is generated by the upper wall of the channel that applies a time-dependent shear stress $S f(t)$ to the fluid. Consequently, contrary to the similar results from the existing literature, the force with which the plate was moved is prescribed and an initially and mixed boundary value problem has been obtained. General expressions for dimensionless velocity and shear stress fields and the adequate frictional force per unit area exerted by the fluid on the stationary plate have been established and the problem under consideration is completely solved. These expressions, that satisfy all imposed initial and boundary conditions, can generate exact solutions for any motion of this kind of respective fluids by choosing adequate forms for the arbitrary function $f(t)$.

For illustration, three motions with engineering applications are considered and some interesting observations and graphical representations are provided and discussed. It is found that the solutions corresponding to motions induced by shear stresses of the form $t^{\alpha} H(t)$ are connected to those of the flow due to a constant shear stress on the boundary by the Riemann-Liouville fractional integral operator $I_{t}^{\alpha}\{\}$ (see Eqs.(3.25)). If $\alpha=n$ (a natural number), these solutions can be obtained by simple or multiple integrations of the above mentioned solutions. Furthermore, in order to be able to determine the required time to reach the steady-state, the solutions corresponding to motions produced by oscillatory or constant shear stresses on the boundary are presented as sums of steady-state and transient components. It is worth pointing out the fact that the steady shear stress $\tau_{C p}$ given by Eq.(3.19) $)_{2}$ is constant on the whole flow 
domain although the corresponding velocity $v_{C p}(y)$ is a function of $y$. The respective constant is just the constant shear stress applied by the upper plate to the fluid to move it. Finally, for validation, the solutions corresponding to oscillatory motions are presented in two different forms whose equivalence has been graphically proved by Figs 1 .
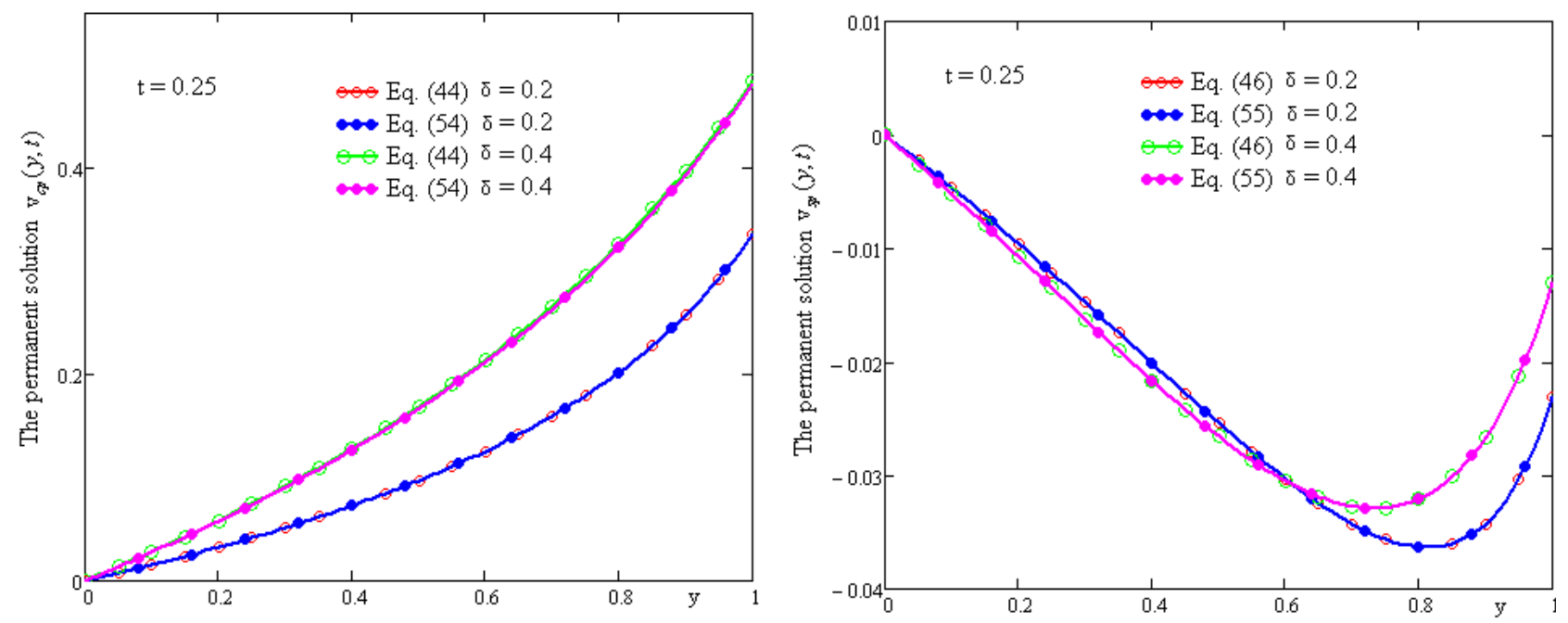

Fig.1. Profiles of the permanent solutions $v_{c p}(y, t)$ and $v_{s p}(y, t)$.

In order to analyze the effects of the shear stress applied by the upper plate on the fluid and the influence of the non-dimensional pressure-viscosity parameter $\delta$ on the dimensionless velocity and shear stress fields, we have considered the case of the oscillating shear stress given by $f(t)=\sin (2 t), t \geq 0$. Numerical results and the corresponding graphical illustrations have been obtained with the software Mathcad using the analytical expressions of velocity and shear stress given by Eqs (3.28) 2 and (3.14).

As observed from Eqs (2.7), (2.8) or (2.15), the studied problem contains as a particular case the Newtonian fluid with constant viscosity; more precisely, when the dimensional pressure-viscosity coefficient $\alpha \rightarrow 0$ (fluid with constant viscosity) and the non-dimensional pressure-viscosity parameter $\delta$ tends to infinity. In this case, the flow of Newtonian fluid with constant viscosity is recovered. The previous observation is illustrated by the graphs from Figs 2 and 3.

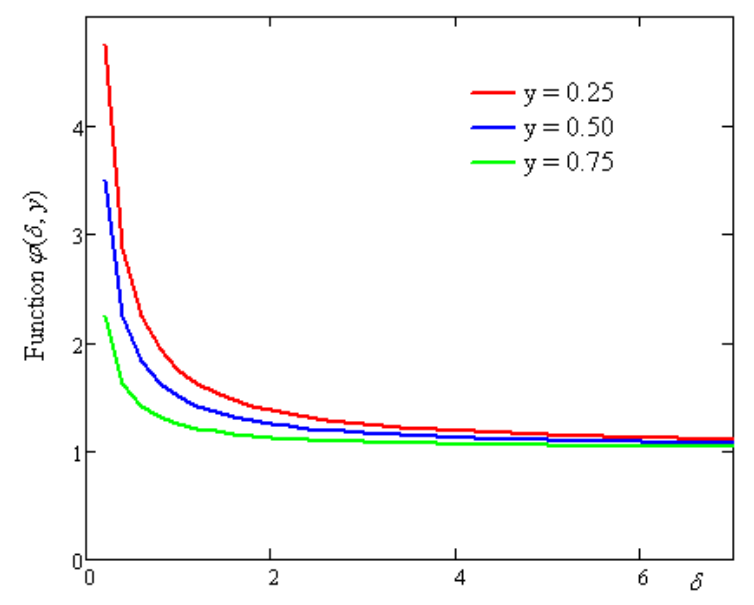

Fig.2. Profiles of the function $\phi(\delta, y)$. 

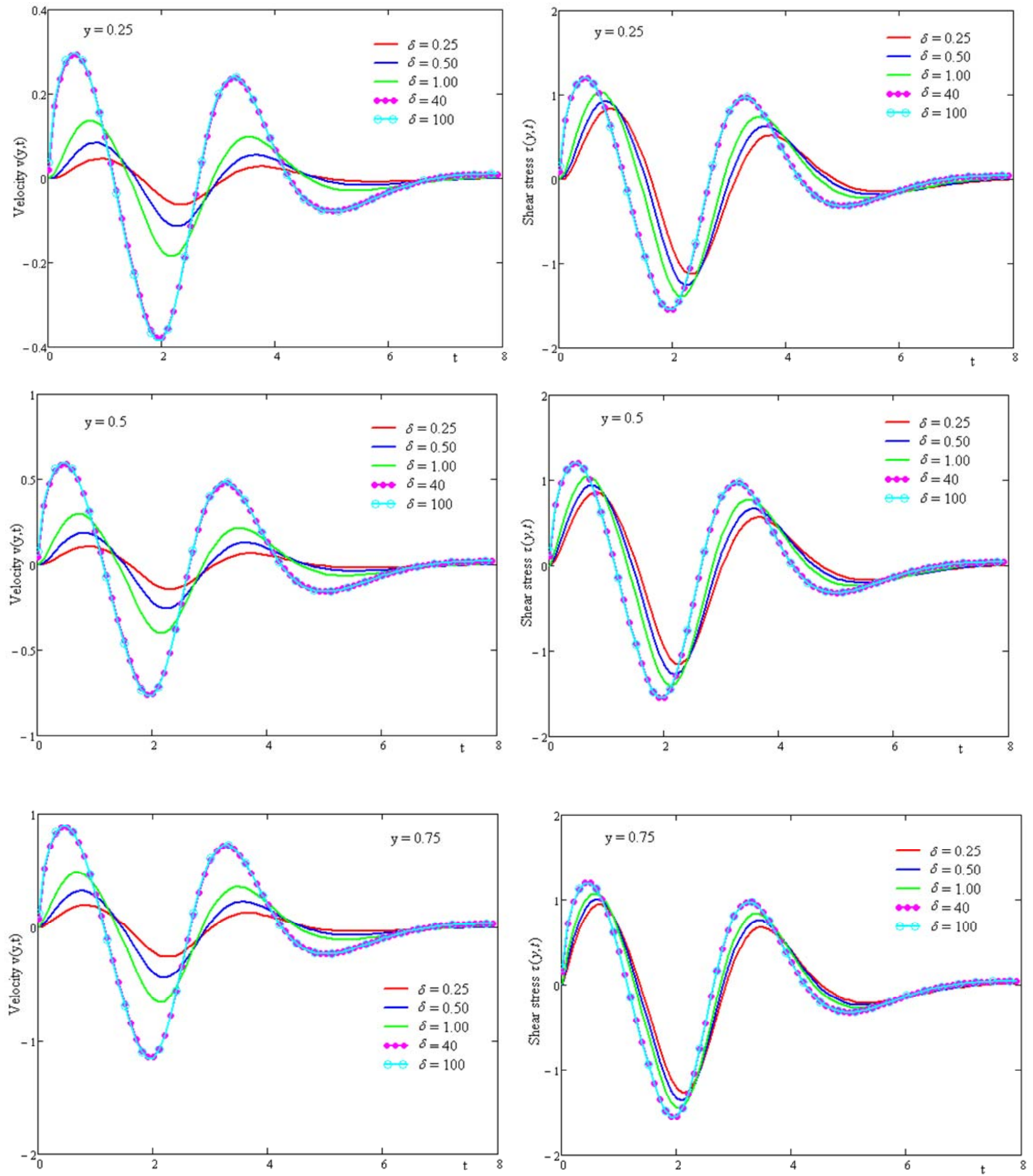

Fig.3. Profiles of the velocity $v_{s}(y, t)$ and shear stress $\tau_{s}(y, t)$ for different values of the parameter $\delta$. 
It is important to note that the influence of the variable viscosity on the non-dimensional shear rate $\frac{\partial v(y, t)}{\partial y}$ is given by the function $\phi(\delta, y)=\frac{\delta+1-y}{\delta}, y \in(0,1), \delta>0$ (see Eq.(2.15), whose profiles are presented in Fig.2. It is observed from the expression of $\phi(\delta, y)$ and from Fig.2 that for large values of the pressure-viscosity parameter $\delta$, the viscosity function tends to 1 for all values of the spatial variable $y$. Also, it should be noted that the fluid viscosity decreases with the spatial position $y$, therefore, the fluid flows slower near the bottom plate and faster near the upper wall. These aspects can be easily observed in Fig.3 which was sketched to show profiles of the velocity $v_{s}(y, t)$ and the shear stress $\tau_{s}(y, t)$, versus the time $t$, for three positions of the channel $y \in\{0.25,0.50,0.75\}$ and for five values of the non-dimensional pressureviscosity parameter $\delta \in\{0.25,0.50,1,1.40,100\}$.

The spatial profiles of the dimensionless velocity and shear stress fields $v_{s}(y, t)$ and $\tau_{s}(y, t)$ have been also plotted in Figs 4-7 for $(y, t) \in[0.1] \times[0.8]$ and for the non-dimensional pressure-viscosity parameter $\delta=0.5$. Some curves obtained by the intersection of these surfaces with planes $y=$ const., respectively $t=$ const. are also presented in these figures. It is observed that the fluid velocity, as well as the shear stress, in absolute value increase with the spatial coordinate $y$. For large values of the time $t$, the fluid has the velocity given by the post-transition solution (the permanent solution). The amplitude of the shear stress with the spatial variable is greater than that of the fluid velocity. In both cases it decreases in time and, as expected, tends to zero for $t \rightarrow \infty$.
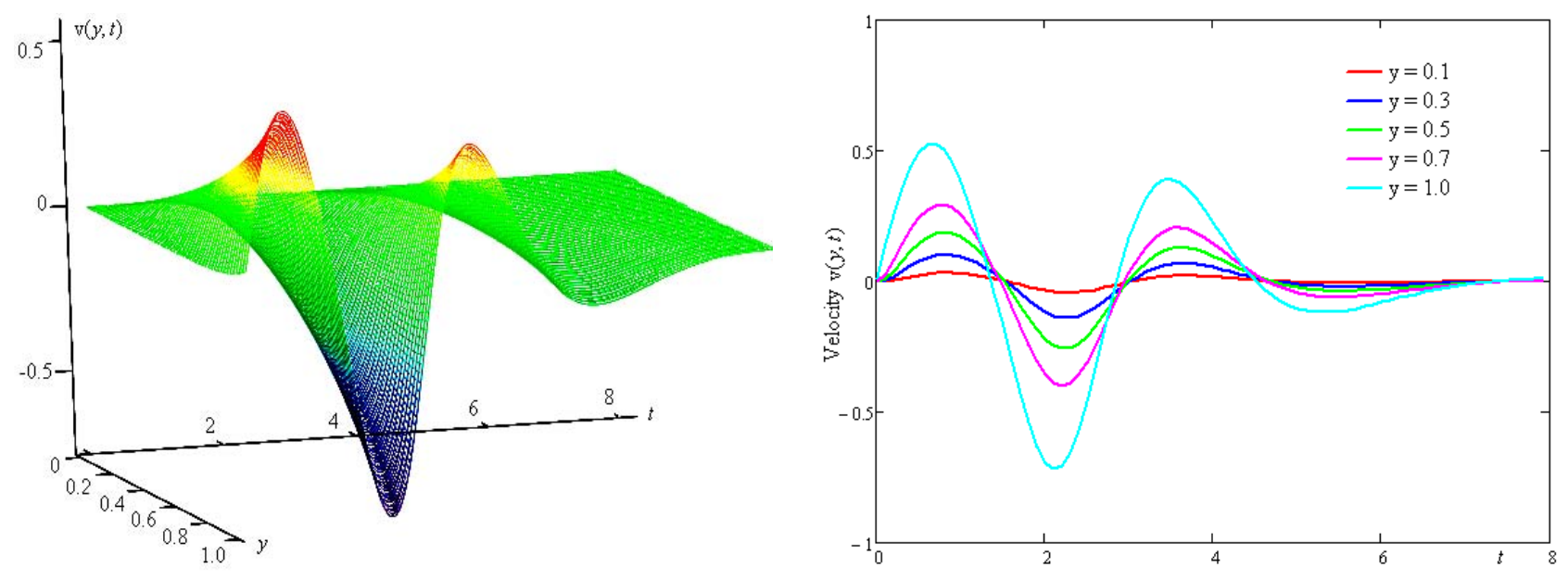

Fig.4. The spatial profile of the velocity $v_{s}(y, t)$ and some sections with planes $y=$ const. 

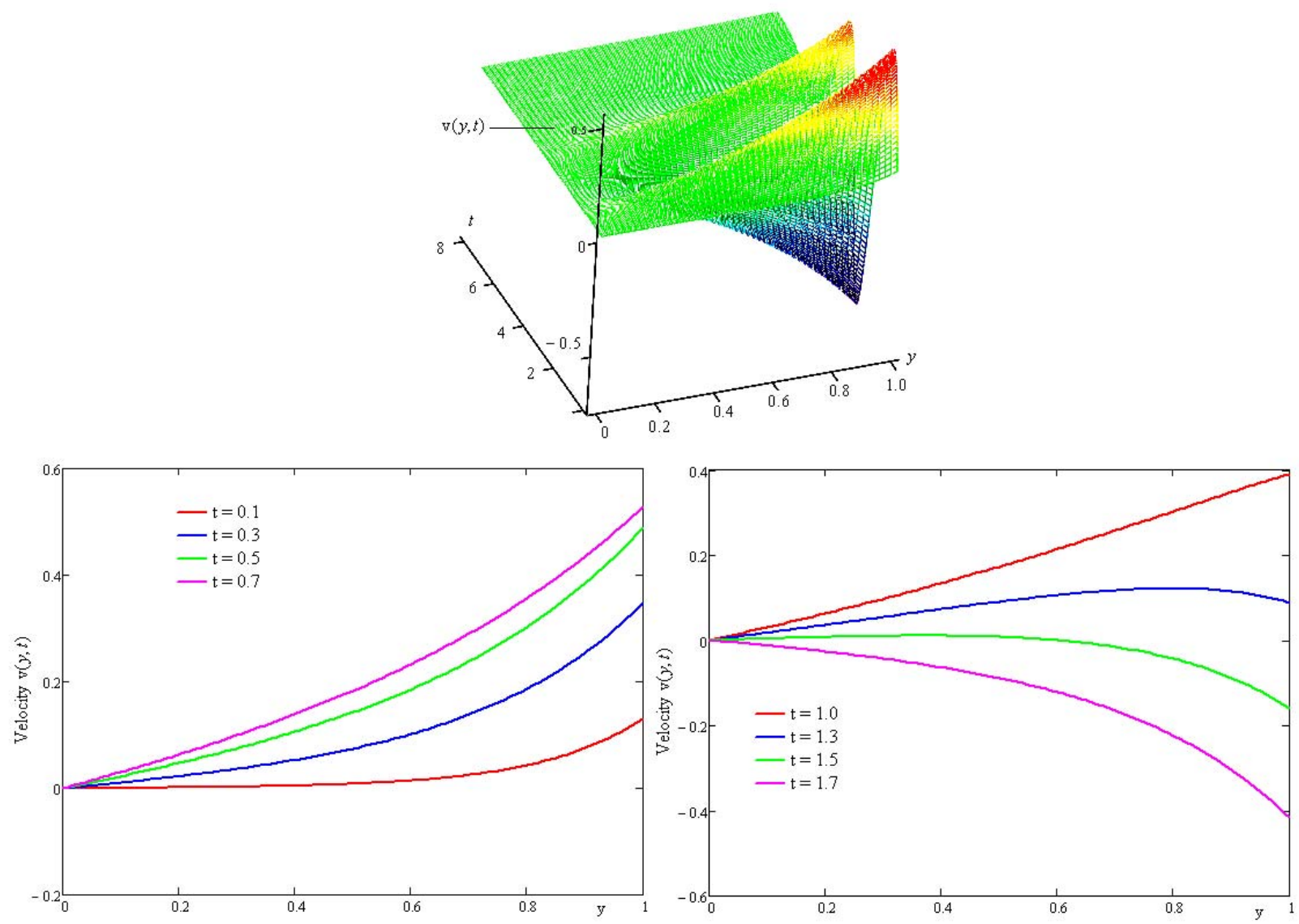

Fig.5. The spatial profile of the velocity $v_{s}(y, t)$ and some sections with planes $t=$ const.
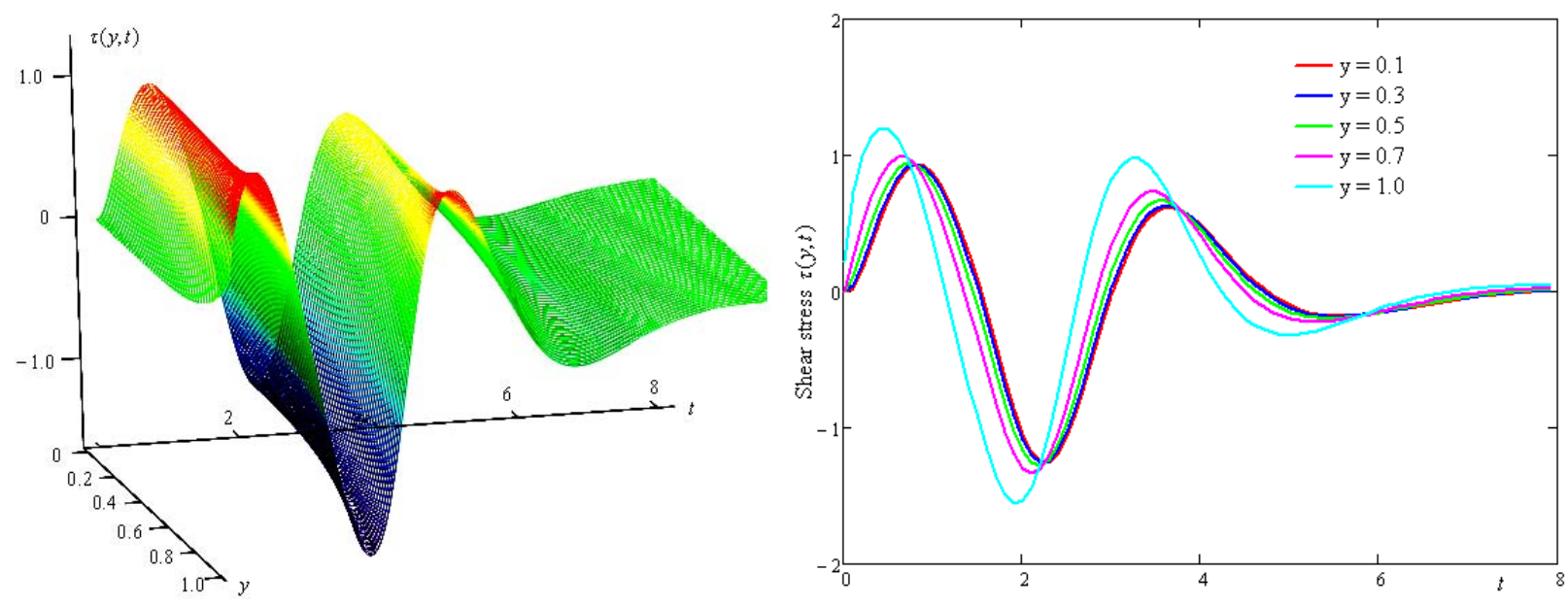

Fig.6. The spatial profile of the shear stress $\tau_{s}(y, t)$ and some sections with planes $y=$ const. 

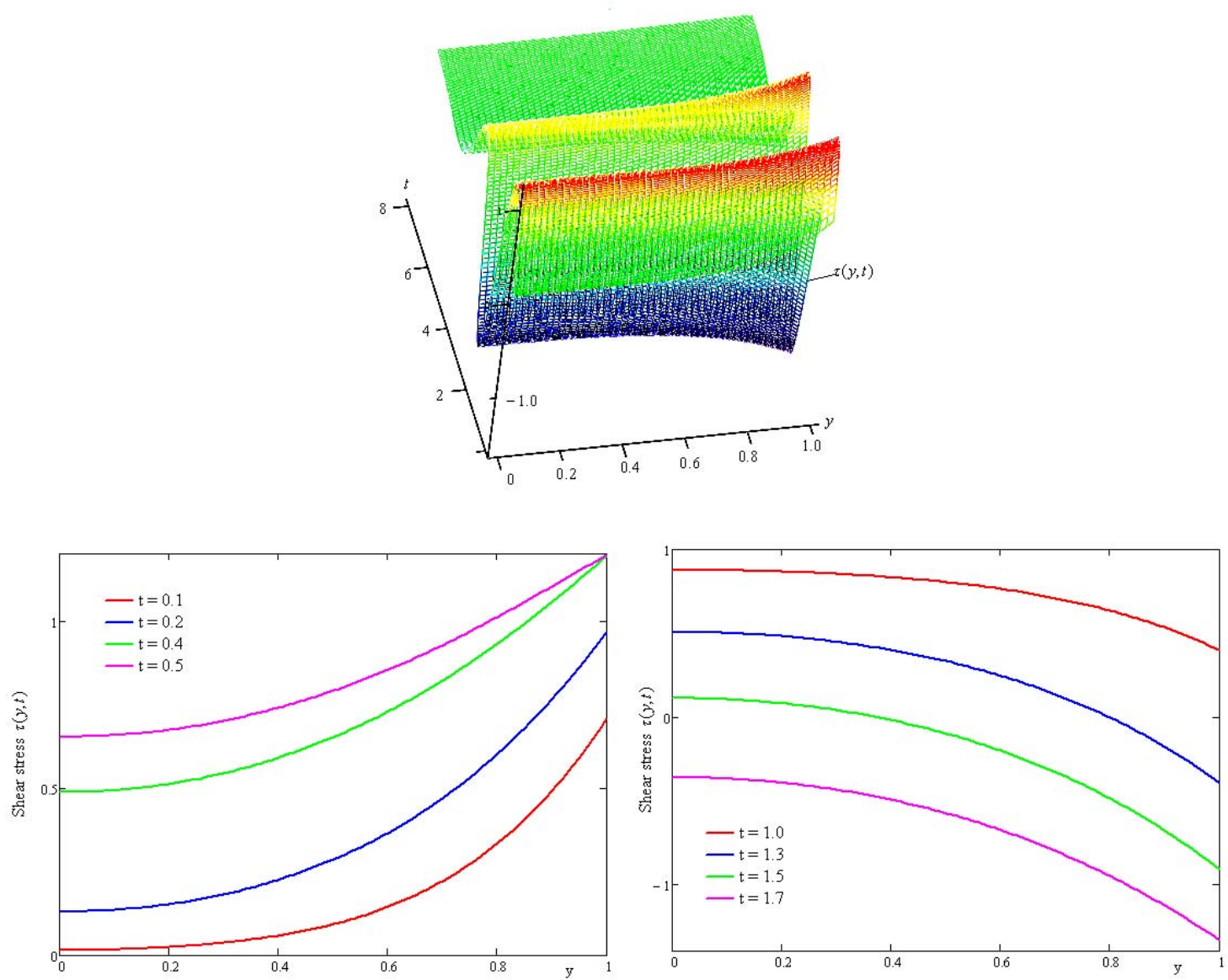

Fig.7. The spatial profile of the shear stress $\tau_{s}(y, t)$ and some sections with planes $t=$ const.

From Figs 4 and 6 it clearly results that the fluid velocity satisfies both the initial condition (2.13) and the boundary condition (2.14) . The shear stress, as it results from Figs 5 and 7 , is also zero for $t=0$ and $y \in(0,1)$. Furthermore, from these figures we can come to the conclusion that, in the analyzed case, after $t=6$ the flow is described by the post-transient solutions as the transient components become negligible after this time.

\section{Appendix}

$$
\begin{aligned}
& \int_{a}^{b} r\left[g^{\prime \prime}(r)+\frac{1}{r} g^{\prime}(r)\right] A\left(r, r_{n}\right) d r=b g^{\prime}(b) A\left(b, r_{n}\right)-a g^{\prime}(a) A\left(a, r_{n}\right)+ \\
& +b r_{n}\left[J_{l}\left(b r_{n}\right) Y_{l}\left(a r_{n}\right)-J_{l}\left(a r_{n}\right) Y_{l}\left(b r_{n}\right)\right] g(b)-r_{n}^{2} g_{H n}, \\
& A\left(a, r_{n}\right)=J_{0}\left(a r_{n}\right) Y_{l}\left(a r_{n}\right)-Y_{0}\left(a r_{n}\right) J_{l}\left(a r_{n}\right)=-\frac{2}{\pi a r_{n}}, \\
& g(r)=\frac{\pi^{2}}{2} \sum_{n=1}^{\infty} \frac{r_{n}^{2} J_{0}^{2}\left(b r_{n}\right)}{J_{l}^{2}\left(a r_{n}\right)-J_{0}^{2}\left(b r_{n}\right)} A\left(r, r_{n}\right) g_{H n} .
\end{aligned}
$$




\title{
Nomenclature
}

\author{
$g$ - acceleration due to gravity \\ $h$ - distance between the plates \\ $p$ - hydrostatic pressure \\ $\boldsymbol{T}$ - Cauchy stress tensor \\ $\boldsymbol{V}$ - velocity vector \\ $\alpha$ - dimensional pressure-viscosity coefficient \\ $\delta$ - non-dimensional pressure-viscosity parameter \\ $\rho$ - fluid density \\ $\mu-$ dynamic viscosity \\ $\frac{\partial}{\partial t}, \frac{\partial}{\partial x}-$ partial derivatives
}

\section{References}

[1] Stokes G.G. (1845): On the theories of the internal friction of fluids in motion, and motion of elastic solids. - Trans. Camb. Phil. Soc., vol.8, pp.287-305.

[2] Bridgman P.W. (1931): The Physics of High Pressure. - Macmillan.

[3] Hron J., Malek J. and Rajagopal K.R. (2001): Simple flows of fluids with pressure dependent viscosities. - Proc. Roy. Soc., London, Ser. A, Mathematical Physical and Engineering Sciences, vol.457, pp.1603-1622.

[4] Rajagopal K.R. and Szeri A.Z. (2003): On an inconsistency in the derivation of the equations of elastodynamic lubrication. - Proc. Roy. Soc., London, Ser. A, vol.459, pp.2771-2786.

[5] Vasudevaiah M. and Rajagopal K.R. (2005): On fully developed flows of fluids with a pressure dependent viscosity in a pipe. - Application of Mathematics, vol.50, pp.341-353.

[6] Rajagopal K.R. (2004): Couette flows of fluids with pressure dependent viscosity. - Int. J. of Applied Mechanics and Engineering, vol.9, No.3, pp.573-585.

[7] Kannan K. and Rajagopal K.R. (2005): Flows of fluids with pressure dependent viscosities between rotating parallel plates. - In: P. Fergola et al. (Eds.), New Trends in Mathematical Physics, World Scientific, Singapore, pp.172183.

[8] Rajagopal K.R. (2008): A semi-inverse problem of flows of fluids with pressure-dependent viscosities. - Inverse Problems in Science and Engineering, vol.16, No.3, pp.269-280.

[9] Massoudi M. and Phuoc T.X. (2006): Unsteady shear flow of fluids with pressure-dependent viscosity. - Int. J. Eng. Sci., vol.44, pp.915-926.

[10] Srinivasan S. and Rajagopal K.R. (2009): Study of a variant of Stokes' first and second problems for fluids with pressure dependent viscosities. - Int. J. Eng. Sci., vol.47, pp.1357-1366.

[11] Rajagopal K.R. and Saccomandi G. (2006): Unsteady exact solution for flows of fluids with pressure-dependent viscosities. - Mathematical Proceedings of the Royal Irish Academy, vol.106A, No.2, pp.115-130.

[12] Prusa V. (2010): Revisiting Stokes first and second problems for fluids with pressure-dependent viscosities. - Int. J. Eng. Sci., vol.48, pp.2054-2065.

[13] Rajagopal K.R., Saccomandi G. and Vergori L. (2013): Unsteady flows of fluids with pressure dependent viscosity. - J. Math. Anal. Appl., vol.404, pp.362-372.

[14] Kalogirou A., Poyiadji S. and Georgiou G.C. (2011): Incompressible Poiseuille flows of Newtonian liquids with a pressure-dependent viscosity. - J. Non-Newtonian Fluid Mech., vol.166, pp.413-419.

[15] Housiadas K.D., Georgiou G.C. and Tanner R.I. (2015): A note on the unbounded creeping flow past a sphere for Newtonian fluids with pressure-dependent viscosity. - Int. J. Eng. Sci., vol.86, pp.1-9. 
[16] Renardy M. (1988): Inflow boundary condition for steady flow of viscoelastic fluids with differential constitutive laws. - Rocky Mountain Journal of Mathematics, vol.18, pp.445-453.

[17] Renardy M. (1990): An alternative approach to inflow boundary conditions for Maxwell fluids in three space dimensions. - J. Non-Newtonian Fluid Mech., vol.36, pp.419-425.

[18] Waters N.D. and King M.J. (1970): Unsteady flow of an elastico-viscous liquid. - Rheol. Acta, vol.9, No.3, pp.345-355.

[19] Debnath L. and Bhatta D. (2007): Integral Transforms and Their Applications, Second Ed. - Chapman and Hall/CRC Press, Boca Raton.

[20] Toki C.J. and Tokis J.N. (2007): Exact solutions for unsteady free convection flows on a porous plate with timedependent heating - Z. Angew. Math. Mech., vol.87, No.1, pp.4-13.

[21] Fetecau C. and Agop M.: Exact solutions for unsteady motion between parallel plates of some fluids with powerlow dependence of viscosity on the pressure. - Sent for publication to Ann. Acad. Rom. Sci. Ser. Math. Appl.

[22] Hristov J. (2019): Linear viscoelastic responses and constitutive equations in terms of fractional operators with non-singular kernels. - Eur. Phys. J. Plus., vol.134, pp.293-323.

Received: November 4, 2019

Revised: March 3, 2020 